\title{
EIRP Characterization of Electrically Large Wireless Equipment with Integrated Signal Generator in a Compact Environment
}

\author{
Soon-Soo Oh, ${ }^{1}$ Woo-Su Kim, ${ }^{2}$ and Young-Hwan Lee ${ }^{3}$ \\ ${ }^{1}$ Department of Electrical Engineering, Chosun University, 309 Pilmun-daero, Dong-gu, Gwangju 501-759, Republic of Korea \\ ${ }^{2}$ Plan and Budget Team, KEIIT, 305 Teheran-ro, Gangnam-gu, Seoul 135-080, Republic of Korea \\ ${ }^{3}$ Radio Technology Research Department, ETRI, 218 Gajeong-ro, Yuseong-gu, Daejeon 305-700, Republic of Korea
}

Correspondence should be addressed to Woo-Su Kim; kws@keit.re.kr

Received 25 August 2014; Revised 26 March 2015; Accepted 31 March 2015

Academic Editor: Ding-Bing Lin

Copyright (C) 2015 Soon-Soo Oh et al. This is an open access article distributed under the Creative Commons Attribution License, which permits unrestricted use, distribution, and reproduction in any medium, provided the original work is properly cited.

\begin{abstract}
We describe a measurement technique to characterize the equivalent isotropically radiated power (EIRP) of electrically large wireless equipment in a compact environment. A modified phase-measurement method was proposed and, thus, the separation of the signal generator and radiating element was not required during the measurement. A Fresnel-to-far-field transformation was used for the fast measurement time in a compact anechoic chamber. An experimental verification of the method was carried out in a compact anechoic chamber, where the source-detector separation was approximately $1 / 5$ of the far-field distance. The measured magnitude and phase pattern exhibited only a small error. The EIRP obtained using a Fresnel-to-far-field transformation was compared with a reference value, and the error was within $0.5 \mathrm{~dB}$.
\end{abstract}

\section{Introduction}

As demand for wireless communication services with low radiation power grows, the restrictions of high-power radiation devices and facilities, such as base stations, satellite stations, and digital broadcasting stations, are becoming increasingly important [1]. Currently, equivalent isotropically radiated power (EIRP) [2] is used as a measure to restrict the radiated power from microwave devices and base stations $[3,4]$.

The EIRP of electrically large wireless equipment is typically characterized using a direct far-field measurement system [5]. The equipment under test (EUT) and probe antenna are separated by a distance $R_{\mathrm{far}}$ that should satisfy Rayleigh's far-field criterion; that is, $R_{\mathrm{far}} \geq 2 D^{2} / \lambda$, where $D$ is the largest dimension of the radiating aperture and $\lambda$ is the wavelength $[2,5]$. Electrically large wireless equipment typically has a farfield distance of more than a few tens of meters. If Rayleigh's criterion cannot be satisfied due to the small size of the anechoic chamber used to characterize the equipment, then the EIRP measurement will be inaccurate [5].

The near-field [6] and Fresnel field scanning methods [7-9] can be used to overcome this limitation. The Fresnel field scanning method has recently been developed and reported to be faster than near-field scanning method [710]. This method requires that both magnitude and phase be recorded; however, it is not straightforward to measure the phase because the signal source is typically contained within the EUT. In some structures, the signal generator cannot be disconnected from the radiating part [11].

The most accurate method is to directly measure the phase information using hardware configuration. The direct method uses the reference signal coupled from the inner circuit of antenna under test (AUT) [5]. The reference antenna method compares the received signals from a reference antenna and the AUT so that the AUT port should be separated [5]. Therefore, two methods cannot be applied to the wireless equipment with integrated signal generator.

Here, we propose an EIRP measurement method that is appropriate for characterizing electrically large EUTs in a compact environment. The EUT can be measured without disconnecting the generator and the radiating element by using the proposed phase-measurement method. The basic theory and the hardware configuration are described, and an experimental verification of the phase pattern and EIRP is provided. 


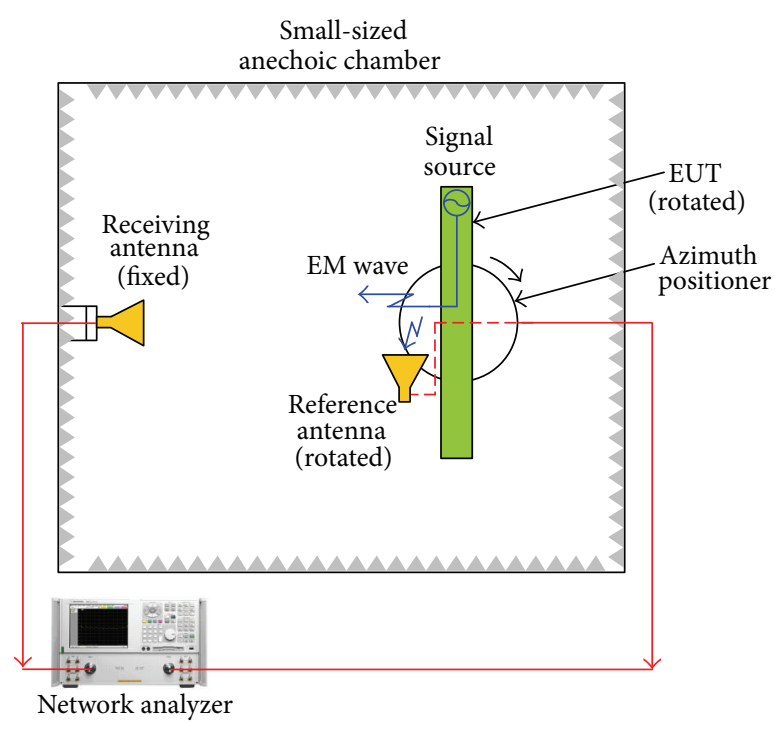

(a)

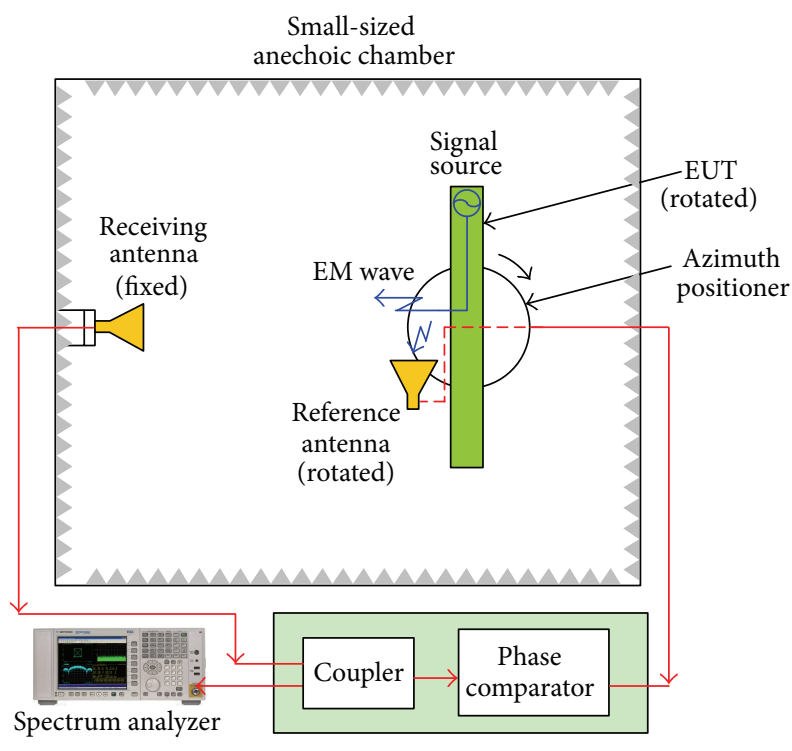

(b)

FIGURE 1: Configuration of the simultaneous phase and magnitude measurement system (a) using a network analyzer and (b) using a spectrum analyzer and phase comparator.

\section{Measurement Method}

2.1. Fresnel-to-Far-Field Transformation. In this section, the Fresnel-to-far-field transformation is briefly discussed. An antenna with aperture dimensions of $L_{x} \times L_{y}$ is assumed to be located at the origin. The electric field in the far-field region $E_{\text {far }}$ is related to the electric field in the Fresnel region $E_{R}$ at a distance $R$, and it can be calculated using the following expression [7-9]:

$$
E_{\mathrm{far}}(\alpha, \beta)=\sum_{m=-M}^{+M} \sum_{n=-N}^{+N} k_{m n} E_{R}(\alpha+m \Delta \alpha, \beta+n \Delta \beta),
$$

where $\Delta \alpha=\lambda / L_{x}$ and $\Delta \beta=\lambda / L_{y}$. Here, the angles $\alpha$ and $\beta$ are in the $y-z$ and $x-z$ planes, respectively. The term $(\alpha+m \Delta \alpha, \beta+n \Delta \beta)$ corresponds to the position to be scanned and is offset from $\alpha$ and $\beta$ by $m \Delta \alpha$ and $n \Delta \beta$, respectively. The coefficient $k_{m n}$ is given by

$$
\begin{aligned}
k_{m n}= & \frac{1}{L_{x} L_{y}} \int_{-L_{x} / 2}^{+L_{x} / 2} e^{+i c^{2} u^{2}} e^{-i\left(2 \pi / L_{x}\right) m u} d u \\
& \cdot \int_{-L_{y} / 2}^{+L_{y} / 2} e^{+i c^{2} v^{2}} e^{-i\left(2 \pi / L_{y}\right) m v} d v,
\end{aligned}
$$

where $c^{2}=\pi /(\lambda R)$.

2.2. Proposed Phase-Measurement Method. In (1), the electric field in the Fresnel region $E_{R}$ is complex, so phase information is required. Figure 1 shows the proposed configuration, in this paper, used to measure the phase pattern. The wireless equipment contains a signal source, and we assume that the signal generator and the antenna element cannot be separated. The receiving antenna receives the emitted electromagnetic (EM) radiation, which is coupled to a network analyzer (NA). Here, the NA is used as a two-channel receiver, so the magnitude of the received power can be measured.

Because the wireless equipment is to be evaluated, it is rotated using the azimuth positioner. The reference antenna is attached to the azimuth positioner and is therefore rotated together with the EUT. Since the receiving antenna is fixed, the reference antenna is moved with EUT so the relative phase difference between the receiving antenna and reference can be measured.

The phase comparison technique is already known, and the reference probe was used in [11] but it was not rotated and applied for the printed circuit board (PCB) scanning. So the configuration shown in Figure 1 has not previously been reported to the best of our knowledge.

As shown in Figure 1(b), a spectrum analyzer with a phase comparator may be used instead of network analyzer. Here, the received signal is coupled to the spectrum analyzer by the coupler.

2.3. EIRP Measurement. Because the electrically large EUT is to be evaluated in a small anechoic chamber, a transformation from the Fresnel field to the far field is used. The procedure is as follows.

(1) Set up the EUT, receiving antenna, and reference antenna, as shown in Figures 1(a) and 1(b).

(2) Set up the instrument to measure the magnitude and phase from the receiving antenna and reference antenna.

(3) Acquire the magnitude and phase of the Fresnel field using an appropriate scanning method.

(4) Transform the Fresnel field to the far field, resulting in the transformed received power $P_{r \text {,transformed }}$. 


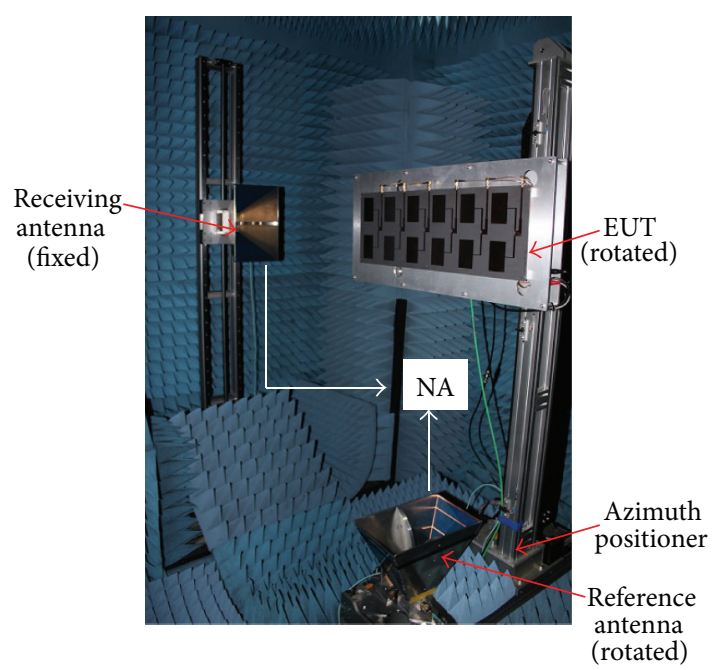

Figure 2: The experimental setup in the anechoic chamber with a range of $1 \mathrm{~m}$.

(5) Calculate the EIRP using the following relation [2]:

$$
\mathrm{EIRP}=\frac{P_{r, \text { transformed }}}{G_{r}} \times\left(\frac{4 \pi R}{\lambda}\right)^{2},
$$

where $G_{r}$ is the gain of the receiving antenna and $R$ is the separation between the EUT and the receiving antenna. One can note that the terms $G_{r}$ and $R$ are directly related to the EIRP, so accurate measures of $G_{r}$ and $R$ are required.

\section{Experimental Results}

3.1. Hardware Configuration. The method was verified via the experimental setup as shown in Figure 2. The frequency was $2.4 \mathrm{GHz}$, and, for simplicity, we used the method with the NA as illustrated in Figure 1(a). A $6 \times 2$ microstrip patch antenna was used as the EUT, and this was connected to a stand-alone signal generator and RF amplifier via a Rotman lens. Although the method was developed for wireless equipment containing a signal generator, we used a stand-alone signal generator to achieve an accurate comparison between the Fresnel transformation and far-field measurements. The patch size was $180 \mathrm{~mm} \times 520.5 \mathrm{~mm}$, resulting in a far-field distance of $R_{\mathrm{far}}=4.9 \mathrm{~m}$.

A double-ridged horn antenna was used as the receiving antenna, which was placed in front of the EUT and connected to the NA. The distance between the EUT and the receiving antenna was $1.0 \mathrm{~m}$, which is approximately $1 / 5$ of the far-field distance.

As shown in Figure 2, a second double-ridged horn antenna was used as the reference antenna. This was placed close to the EUT, and the signal from the reference antenna was also fed into the NA. Because the reference antenna was fixed on the azimuth positioner, it was rotated with the EUT, as described in the previous section. Therefore, the phase difference between EUT and reference antenna remained constant as the azimuth positioner rotated the EUT.

During the measurement of the magnitude and the phase patterns of EUT, the NA was used as a two-channel receiver. Although the setup for this is dependent on the NA model, the output signal from the port should first be disconnected. The absolute levels of signals $\mathrm{A}$ and $\mathrm{B}$ were compared, resulting in the ratio $A / B$ and the phase pattern, where $A$ is the signal to port 1 and $B$ is the signal to port 2 [12].

3.2. Fresnel Field Pattern and EIRP. The scanned Fresnel fields at $\alpha=0^{\circ}$ are plotted in Figure 3. These were measured by rotating the azimuth positioner at a resolution of $1.0^{\circ}$. Figure 3(a) shows the magnitude pattern, which was obtained using the two-channel receiver mode of the NA as proposed in this paper. The pattern acquired using the spectrum analyzer is also shown with the legend of "Reference." The two sets of magnitude patterns are in very good agreement. One must be careful that Fresnel-to-far-field transformation is only valid in the angular range of -90 to +90 degrees because of the scanning range.

Figure 3(b) shows the phase, which was obtained using the method described in the previous section. In Figure 3(b), the phase pattern acquired using the transmission measurement mode of the NA is also shown with the legend of "Reference." The maximum difference between the two patterns over the range of $180^{\circ}$ was $8.5^{\circ}$.

The calculated EIRP was compared with that measured using a direct far-field method; the results are summarized in Table 1. The far-field data were obtained by disconnecting the generator and EUT. The transmitted power $P_{t}$ from the generator was measured using the spectrum analyzer, and the gain of antenna $G_{t}$ was characterized in an anechoic chamber at a range of $7 \mathrm{~m}$. The EIRP was obtained by summing $P_{t}$ and $G_{t}$ in $\mathrm{dB}$.

The EIRP was obtained using the power from the Fresnelto-far-field transformation using $P_{r}-G_{r}+20 \log \left(4 \pi R_{2} / \lambda\right)$ in $\mathrm{dB}$, where $P_{r}$ is the transformed field, $G_{r}$ is the gain of the receiving antenna, and $R_{2}$ is the far-field distance [2]. The used values of $M$ and $N$ in (1) were 7 and 1, respectively. The EIRP was $21.54 \mathrm{dBm}$, measured using the Fresnel-to-farfield transformation, which was in agreement with the farfield measurement to within $0.5 \mathrm{~dB}$. This error may be further reduced by increasing the number of $\alpha$-plane to be scanned and more precisely calibrating the receiving probe.

\section{Conclusion}

We have described an experimental method to characterize the EIRP of electrically large antennas in a compact environment where the generator and radiating element are not required to be separated. A Fresnel-to-far-field transformation was used, and the phase was obtained by comparing the signal at a receiving antenna and a reference antenna. An experimental verification was performed in a small anechoic chamber at a range of $1 \mathrm{~m}$, which is approximately $1 / 5$ 
TABLE 1: Comparison of the EIRP calculated using the two different methods.

\begin{tabular}{|c|c|c|c|c|c|c|}
\hline \multirow{3}{*}{$\begin{array}{l}\text { Method } \\
\text { Far field (isolating } \\
\text { generator and EUT) }\end{array}$} & \multirow{3}{*}{$\begin{array}{c}\text { Expression } \\
\operatorname{EIRP}[\mathrm{dB}]=P_{t}+G_{t}\end{array}$} & \multicolumn{3}{|c|}{ Power and gain } & \multirow{3}{*}{$\begin{array}{c}\text { EIRP }[\mathrm{dBm}] \\
+21.04\end{array}$} & \multirow{3}{*}{$\begin{array}{c}\text { Error }[\mathrm{dB}] \\
-\end{array}$} \\
\hline & & $P_{t}$ & -11.53 & $\mathrm{dBm}$ & & \\
\hline & & $G_{t}$ & 32.57 & $\mathrm{dBi}$ & & \\
\hline \multirow{4}{*}{$\begin{array}{l}\text { Fresnel-to-far-field } \\
\text { transformation } \\
\text { (proposed) }\end{array}$} & \multirow{4}{*}{$\operatorname{EIRP}[\mathrm{dB}]=P_{r}-G_{r}+20 \log \left(\frac{4 \pi R_{2}}{\lambda}\right)$} & $P_{r}$ & -23.32 & $\mathrm{dBm}$ & \multirow{4}{*}{+21.54} & \multirow{4}{*}{0.5} \\
\hline & & $G_{r}$ & 11.1 & $\mathrm{dBi}$ & & \\
\hline & & $R_{2}$ & 7.0 & $\mathrm{~m}$ & & \\
\hline & & $\lambda$ & 0.14 & $\mathrm{~m}$ & & \\
\hline
\end{tabular}

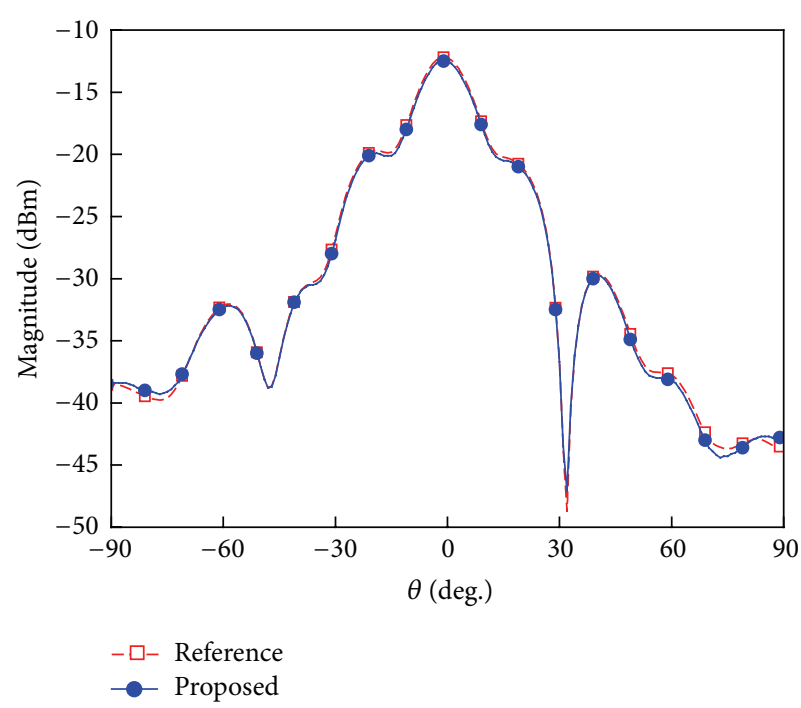

(a)

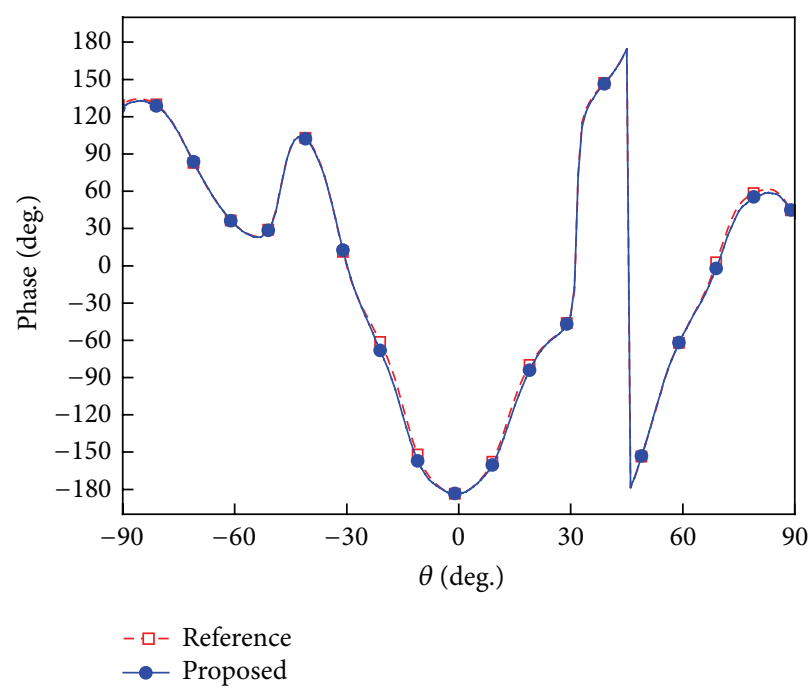

(b)

FIgURE 3: Fresnel fields measured at $2.4 \mathrm{GHz}$ at $1 \mathrm{~m}$ : (a) magnitude and (b) phase.

the far-field distance, and the phase pattern was successfully characterized. We determined the EIRP with an error of $0.5 \mathrm{~dB}$ compared with a direct far-field measurement. This technique is therefore suitable for characterizing large antennas in small anechoic chambers.

\section{Conflict of Interests}

The authors declare that there is no conflict of interests regarding the publication of this paper.

\section{Acknowledgments}

This research was supported by research fund from Chosun University in Korea, 2013, and this work (Grants no. C0221835) was supported by Business for AcademicIndustrial Cooperative Establishments funded Korea Small and Medium Business Administration in 2014.

\section{References}

[1] IEEE Std. 802.16.2-2004, IEEE Recommended Practice for Local and Metropolitan Area Networks. Coexistence of Fixed Broadband Wireless Access Systems, 2004.
[2] W. L. Stutzman and G. A. Thiele, Antenna Theory and Design, John Wiley \& Sons, New York, NY, USA, 1998.

[3] FCC CFR Title 47: 25.218 Off-axis EIRP envelopes for FSS earth station operations, 2009.

[4] R. V. Gatti, L. Marcaccioli, E. Sbarra, and R. Sorrentino, "Flat array antennas for Ku-band mobile satellite terminals," International Journal of Antennas and Propagation, vol. 2009, Article ID 836074, 5 pages, 2009.

[5] J. D. Kraus and R. J. Marhefka, Antennas for All Applications, McGraw-Hill, Boston, Mass, USA, 2002.

[6] IEEE Std 1720-2012, IEEE Recommended Practice for Near-Field Antenna Measurements, IEEE, 2012.

[7] G. E. Evans, "Far field correction for short antenna ranges," in Proceedings of the Antenna Measurement Techniques Association (AMTA '85), pp. 34.1-34.9, 1985.

[8] I. L. Vilenko, A. A. Meduhin, Y. A. Suserov, A. K. Tobolev, and A. V. Shishlov, "Reconstruction of antenna radiation pattern by using data of measurements in a Fresnel region with test facility intended for far-field measurements," Antennas, pp. 4652,2005

[9] S.-S. Oh, J.-M. Kim, and J.-H. Yun, "Antenna measurement on cylindrical surface in Fresnel region using direct far-field measurement system," ETRI Journal, vol. 29, no. 2, pp. 135-142, 2007.

[10] M. Sierra-Castaner and S. Burgos, "Fresnel zone to far field algorithm for rapid array antenna measurements," in Proceedings of 
the 5th European Conference on Antennas and Propagation, pp. 3251-3255, April 2011.

[11] S.-H. Yan and T.-H. Chu, "A beam-steering and -switching antenna array using a coupled phase-locked loop array," IEEE Transactions on Antennas and Propagation, vol. 57, no. 3, pp. 638-644, 2009.

[12] Y. Vives-Gilabert, C. Arcambal, A. Louis, F. de Daran, P. Eudeline, and B. Mazari, "Modeling magnetic radiations of electronic circuits using near-field scanning method," IEEE Transactions on Electromagnetic Compatibility, vol. 49, no. 2, pp. 391-400, 2007. 

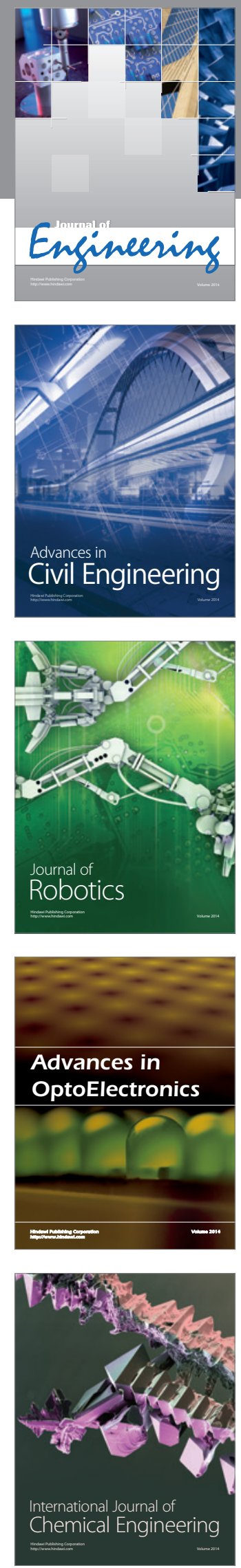

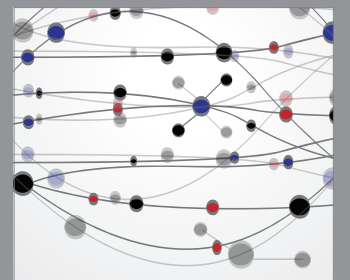

The Scientific World Journal
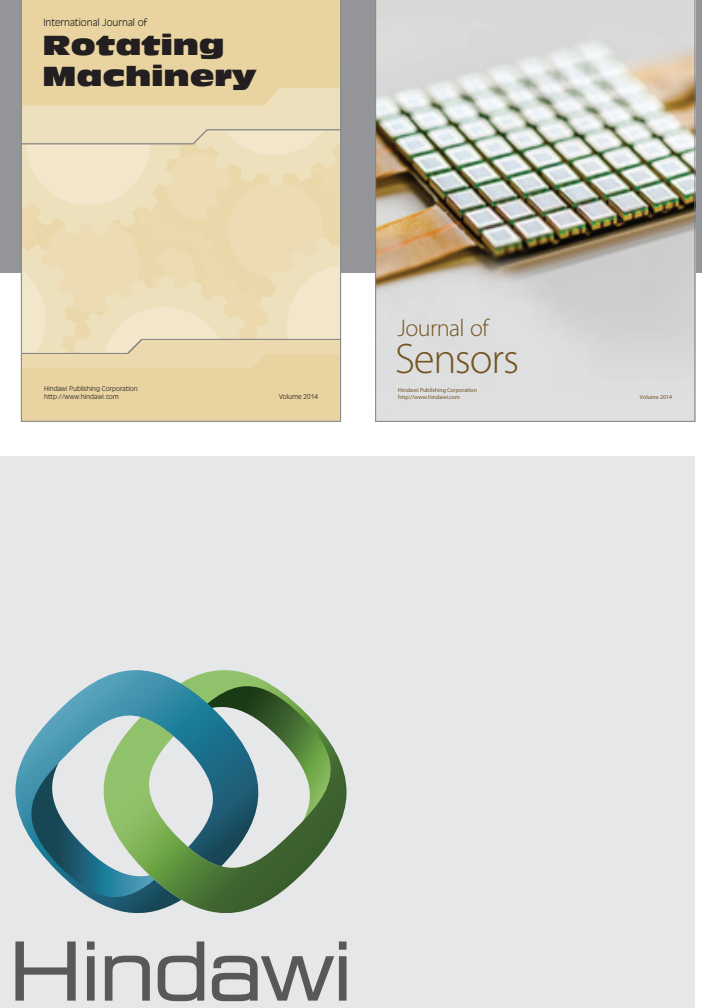

Submit your manuscripts at http://www.hindawi.com
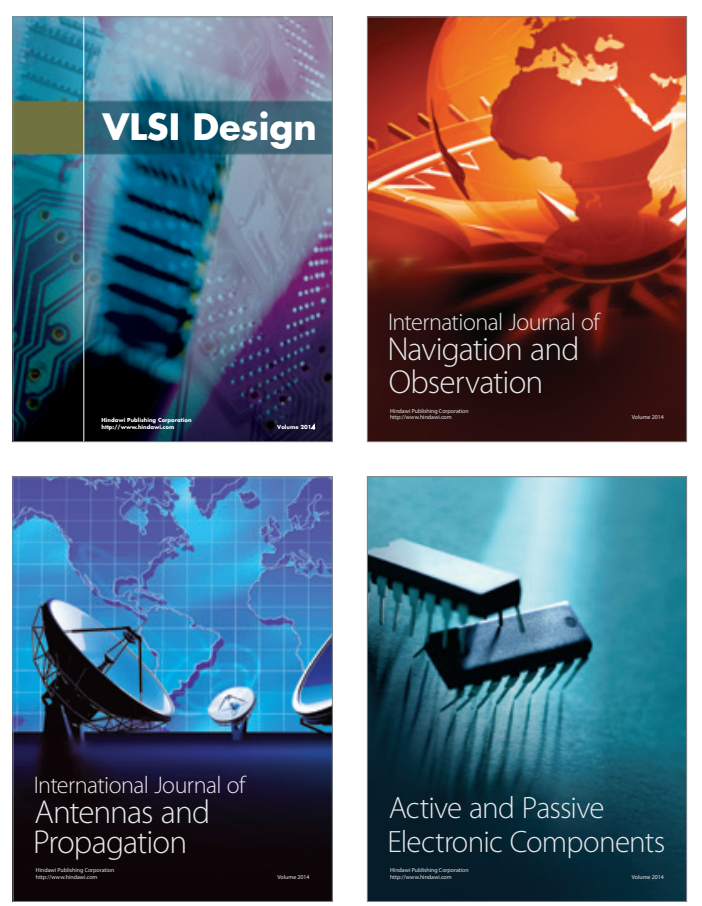
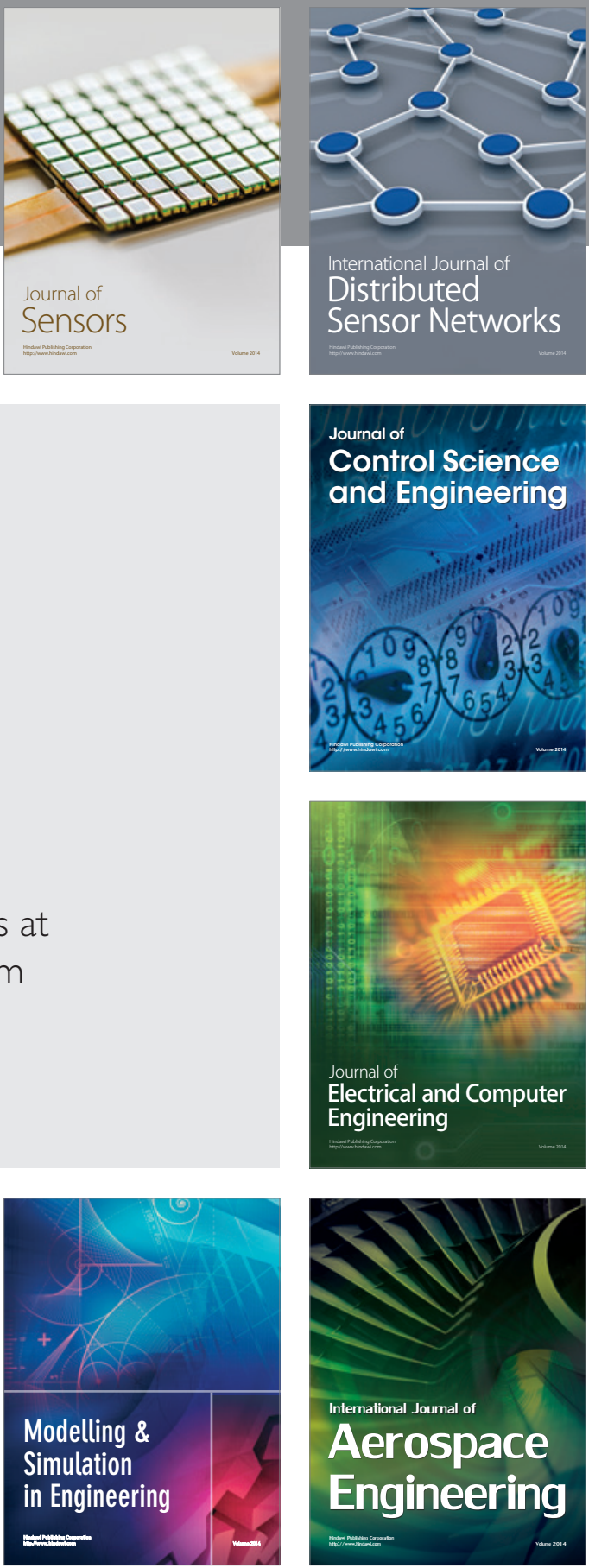

Journal of

Control Science

and Engineering
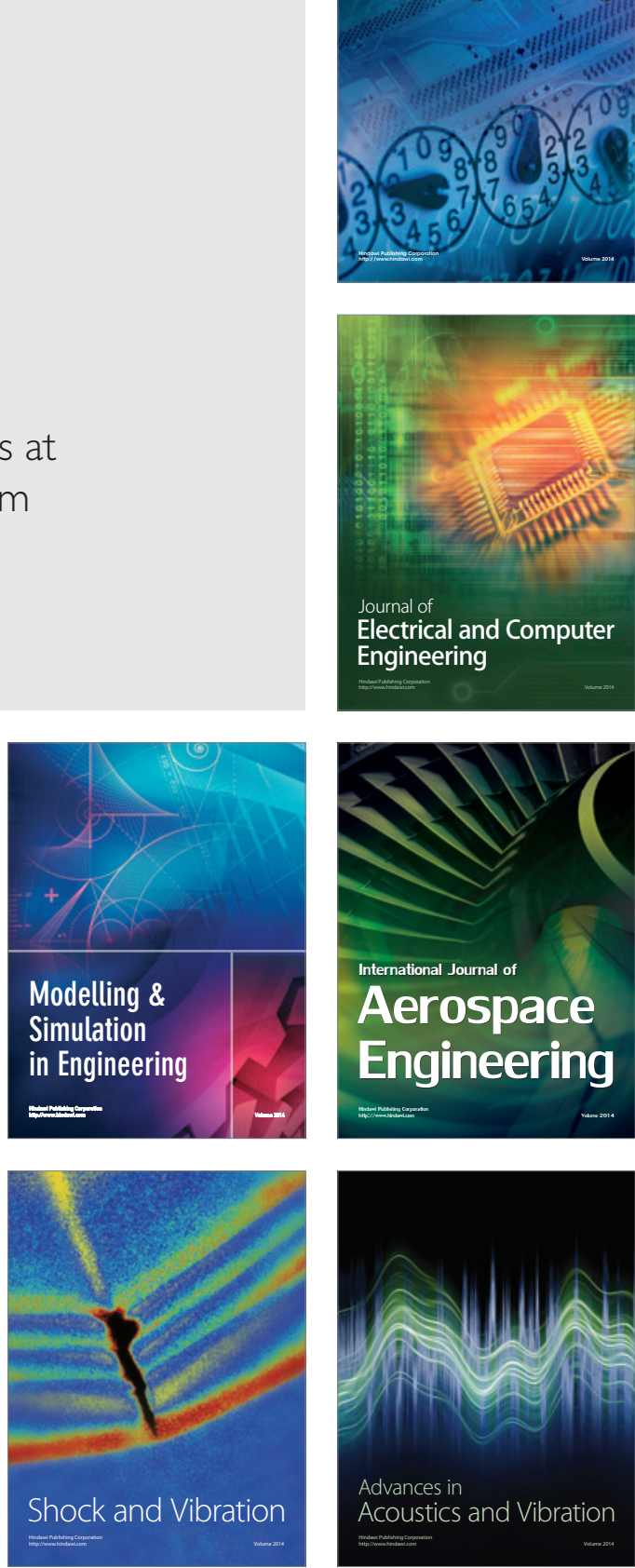